\title{
Eye movements of stumptailed monkeys during discrimination learning: VTE revisited
}

\author{
ALLAN M. SCHRIER and MORRIS L. POVAR \\ Brown University, Providence, Rhode Island 02912
}

\begin{abstract}
Eye movements of stumptailed monkeys were measured during learning of a pattern discrimination problem. Amount of scanning (shifts in visual fixation from one pattern to the other) increased once learning began and reached a maximum either at about the point that frequency of correct responses reached asymptote or just after that. These results, taken together with earlier findings on vicarious trial and error and operant observing behavior, suggest that this is the characteristic pattern of change in frequency of observing during visual discrimination learning. A change in information processing strategy is proposed as one explanation for these results. Duration of the last fixation on a discriminative stimulus during a trial showed similar, though not identical, changes with practice. The decrease in duration of the last fixation following learning contrasts with results of prior studies involving more complex tasks, and supports our conclusion that the duration of the last fixation is particularly sensitive to the cognitive processing requirements of the discriminative task.
\end{abstract}

During the past several years, our laboratory has reported a variety of changes in the ocular observing behavior (that is, number and duration of eye fixations) of monkeys during brightness discrimination reversal learning (Schrier \& Wing, 1973), during the learning of a series of color and form discrimination problems involving reversal and nonreversal shifts (Schrier \& Vaughan, 1973), during learning-set formation over the course of a long series of dot-pattern discrimination problems (Schrier \& Povar, 1978), and as a function of problem complexity (Geary \& Schrier, 1975). We have not yet explored systematically the changes in ocular observing behavior during the original learning of single, simple discrimination problems, although a number of questions have arisen about such changes in the course of our own prior work as well as related work by others.

An older related literature, once representing a very active research area with rats usually the subjects, is that of vicarious trial and error (VTE). This refers to a subject's tendency to vascillate at choice points before the choice response occurs. The behavior measured (such as head turning) was not always equivalent to observing behavior as the term is used today, because it did not always result in ex-

This research was supported by Grant BNS76-00036 from the National Science Foundation. The authors are greatly indebted to both Judith E. Schrier and Claudia R. Thompson for their help in carrying out the research and for their critical readings of the manuscript. Requests for reprints should be addressed to Allan M. Schrier, Psychology Department, Brown University, Providence, Rhode Island 02912. posure of the subject to the discriminative stimuli, but there is no doubt that the two types of behavior have much in common. Tolman $(1938,1948)$ reported that, in general, frequency of VTE increases during the learning of visual discrimination problems. Following a brief period at maximum levels, a decline in VTE was usually reported if the problem was not difficult (Tolman \& Minium, 1942). Muenzinger (1938) also concluded that VTE was most likely to occur on the trials near the point that learning was complete. Other evidence, such as that faster learners do more VTEing (Geier, Levin, \& Tolman, 1941), led both Muenzinger and Tolman to conclude that this behavior facilitates visual discrimination learning.

As Goss and Wischner (1956) pointed out in a review of the topic, the difficulty of measuring such behaviors as rapid head turning led many investigators to report only the percentage of trials on which VTE occurred, rather than its frequency of occurrence on each trial. It appears that the increasingdecreasing VTE function described above was more likely to be reported with the latter measure than with the former. This, together with the variations in measures of VTE and the tendency to lump results of maze and visual discrimination learning studies together, has made the VTE findings appear more chaotic than they are.

Another related literature, reflecting a currently active research area, is that dealing with nonocular visual observing behavior (that is, operant responses which produce the discriminative stimuli, e.g., Wyckoff, 1952). Most of these studies have been 
carried out in operant contexts and are not of immediate concern here. One, however, by D'Amato, Etkin, and Fazzaro (1968), is of particular relevance because it involved measurement of nonocular observing responses by cebus monkeys during discrete trial, two-choice simultaneous discrimination learning, which is the typical procedure in our ocular observing response studies. The changes in frequency of observing responses by the two animals for whom data on original learning of a discrimination problem are presented are quite similar to those described for VTE, there having been an increase and then a decrease with the maximum frequency occurring when learning had reached a high level. It seems possible that this type of curve may be typical for frequency of observing during original learning of visual discriminations, since it was also found for ocular observing (Geary \& Schrier, 1975) during learning of a form discrimination by two stumptailed monkeys.

The purpose of the present experiment was to investigate ocular observing behavior of stumptailed monkeys during the learning of a single, two-choice visual discrimination problem. The two major measures of ocular observing that we used were frequency of scans per trial (number of shifts in fixation from one discriminative stimulus to the other) and duration of each fixation on a discriminative stimulus. The former is a much more accurate measure of VTE than were those in the earlier studies on the question. Duration of individual observing responses has been given almost no attention in the previous literature, although we have found it to be sensitive to a number of variables that either do not influence frequency of scanning or influence it in a different manner (Geary \& Schrier, 1975; Schrier \& Povar, 1978).

\section{METHOD}

\begin{abstract}
Subjects
Eight wild-born male stumptailed monkeys (Macaca arctoides), between 3.5 and 5 years of age, served as the subjects. All animals were housed in individual cages in a colony room where they had free access to water. On test days, each animal received about $50 \%$ of its daily food ration in the form of $190-\mathrm{mg}$ bananaflavored whole-diet pellets (Noyes, Lancaster, N.H.), which were used as reinforcers in the experimental situation, with the balance of the ration, a conventional primate biscuit, fed in the home cages. The animals' weights remained constant or increased very slightly during the course of the experiment.
\end{abstract}

\footnotetext{
Apparatus

The main components of the apparatus, the details of which have been described before (Geary \& Schrier, 1975; Schrier, Povar, \& Vaughan, 1970; Vaughan, 1975), were (1) a restraining chair, (2) a "crown" set on each monkey's head, which could be fastened to the restraining chair in order to immobilize the head, as required by the corneal reflection technique, (3) a stimulus presentation and response panel (response panel) attached to the front of the restraining chair and facing the monkey, (4) a light source for
}

creating a bright corneal reflection and a special TV camera which transmitted the reflection for both visual monitoring and electronic analysis, and (5) a computer and associated interface for recording the location of the corneal reflection and controlling and recording the experimental events.

The response panel contained three 2.5 -cm-diam circular response keys, made of clear plastic with a 9-mm-diam hole in the center of each. The keys were arranged in the form of an inverted triangle, with the one at the bottom referred to as the "center key" and those at the top, the "side keys." The distance between the side keys was $8 \mathrm{~cm}$ center to center (visual angle approximately $15^{\circ}$ ) and that between each side key and the center key was $5.5 \mathrm{~cm}$ (visual angle approximately $10^{\circ}$ ). The discriminative stimuli were displayed on an oscilloscope, the tube face of which was mounted directly behind the three keys in the response panel. A pattern could be created by lighting small dots in a 6 by 6 matrix behind the holes in each of the side keys. Two problems were presented. One was closed vs. open square (squares problem), the sides of each of which were approximately $3 \mathrm{~mm}$ (visual angle approximately $34^{\prime}$ ). The other problem consisted of a pair of irregular patterns of seven dots selected from the set used by Schrier and Povar (1978).

\section{General Procedure}

There was one test session a day, 5 days a week. Each session consisted of 300 experimental trials in addition to the 20 or 30 trials necessary to achieve initial alignment of the corneal reflection equipment. A brightness discrimination problem was presented during the preexperimental trials. The intertrial interval was $5 \mathrm{sec}$. Each trial consisted of two phases, a "calibration" phase and a "discrimination" phase. The calibration phase, which involved only the center key, functioned to allow the experimenter to maintain the equipment in spatial calibration from trial to trial. Most of the details of the calibration phase and the reasons for the procedures were described by Schrier and Vaughan (1973), so only the few changes that were introduced will be mentioned here. The calibration phase began with the illumination of a small dim spot behind the hole in the center key in addition to the onset of the tone used in our prior studies. When the duration of a fixation on or near the spot of light exceeded $.75,1.00,1.25$, or $1.50 \mathrm{sec}$, the specific value for a given trial selected at random from among these, the spot of light brightened for $382 \mathrm{msec}$. If the animal pressed the center key during the bright light period or within about $1 \mathrm{sec}$ of its onset, the discrimination phase was initiated. The latter phase consisted of the presentation of the pattern stimuli behind the side keys and had a maximum duration of $4 \mathrm{sec}$, almost always much more time than any animal needed to make the choice response. Data analysis involved only the discrimination phase, that is, the period from the onset of the discriminative stimuli on the side keys to the occurrence of a choice response. Recorded fixations were defined as being on the discriminative stimuli in much the same way as in the previous research, except that the size of each discriminative stimulus area and its location on the recording grid were adjusted to compensate for minor dayto-day differences in alignment of the recording apparatus.

\section{Pretraining and Experimental Training}

Using standard operant techniques, the animals were pretrained while in the chair to press the response keys under conditions of a brightness discrimination task. Animals $D, F, J, K, L$, and $R$ continued to receive the brightness discrimination task during the final stages of pretraining when the head was restrained and the TV camera and light source for the corneal reflection were moved into place. Animals $M$ and $\mathbf{P}$ were given the squares problem during this period. Pretraining required a mean of 43 sessions.

Following pretraining, animals D, F, J, K, L, and $R$ were given the squares problem, and $M$ and $P$, the irregular-pattern problem, with all the animals except $J$ trained until both correct choice responses and ocular observing behavior appeared to have leveled off. Animal J's crown came loose following the 10th test session 
and testing on the squares problem was not resumed for reasons unrelated to the present experiment.

\section{RESULTS}

Mean number of scans per trial and the percentage of correct responses as a function of days (sessions) of practice are shown in Figure 1 for each of the eight animals. All of the animals showed the largest increase in percentage of correct choice responses during the first 4 days. The asymptotic level of choice performance was above $90 \%$ correct for all animals except $R$ and $L$. The pattern of change in amount of scanning as a function of practice was similar for all animals, with perhaps the exception of animal L, whose performance on the discrimination task was the worst of the group and the most variable. All animals maintained a relatively low level of scanning during the presolution period when performance was at the chance level. Once learning began, there was a sharp increase in amount of scanning from an initial low level to a maximum, and then, typically, after 1 or 2 days at or near the maximum level, a decrease. Except for animal $R$, the terminal levels of scanning were at or close to the minimum level necessary for the animal to observe the positive stimulus before making the choice response. In the present situation, this minimum level is an average of $.5 \mathrm{scans} / \mathrm{trial}$ (Geary \& Schrier, 1975; Schrier \& Wing, 1973).

As a means of testing these observations statistically, the experimental sessions for each animal were divided into six successive blocks: (1) the presolution period when performance was at the chance level, (2) the first and (3) the second half of the testing period which preceded (4) the session during which the maximum amount of scanning occurred, and (5) the first and (6) the second half of the testing period following the session of maximum scanning. The taped data of animal $K$ were unavailable for this analysis. The mean amount of scanning per trial for the remaining seven subjects for each of the six successive blocks was $.27, .38, .52$, $.71, .52$, and .46 , respectively. A Treatments by Subjects analysis of variance indicated that the effect of blocks was highly significant $[F=(5,30) 14.2, p<$ $.001]$. The Newman-Keuls test for differences between pairs of means indicated the following significant differences (ps $<.05$ ): those between Block 4 and all other blocks, those between Block 1 and all other blocks except Block 2, and those between Block 2 and Blocks 5 and 3. All other comparisons were not significant. The mean amount of scanning during Block 6 was not significantly different from .5 , even when the results for animal $K$ (which could be determined for this block from the data available) were substituted for those for the very low scanning animal R. Amount of scanning by this

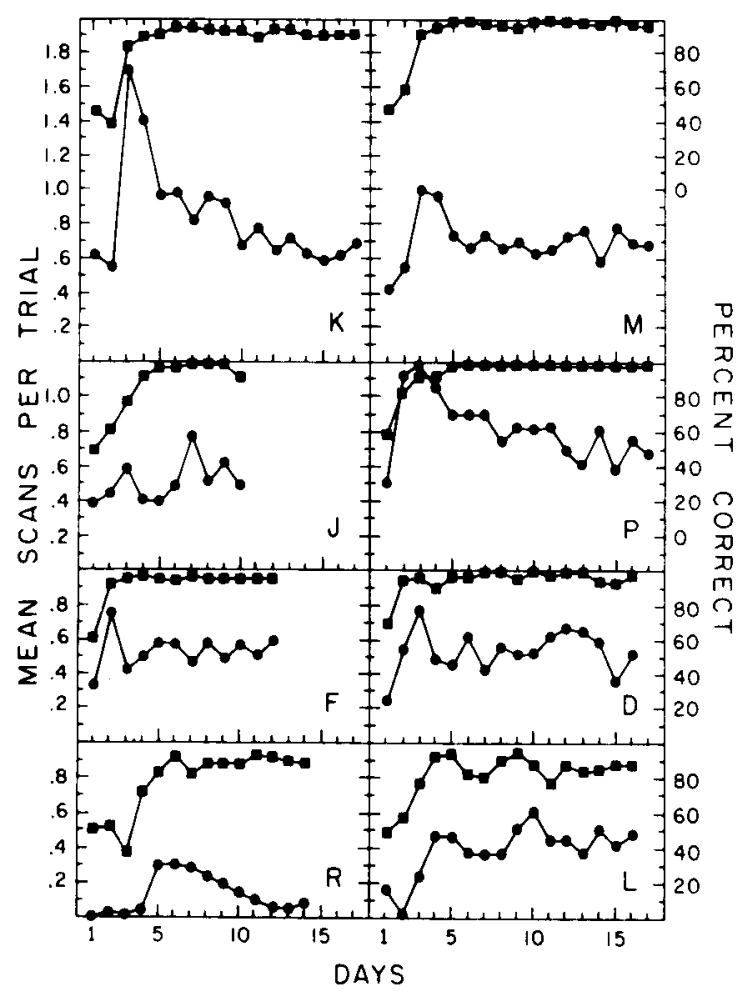

Figure 1. Mean number of scans per trial (circles and left ordinate) and percentage of correct responses (squares and right ordinate) by each of the eight monkeys as a function of days (sessions) of practice. Animals $M$ and $P$ received the irregularpattern problem and the remaining animals the squares problem.

latter animal, which never reached as high a level as that for the other animals, declined to nearly zero. A high performance level despite such a low level of scanning could occur either because the animal observed the correct discriminative stimulus by means of peripheral vision while responding to the center key, or, as was the case here, because it adopted an unusual conditional strategy of responding to the side key it first fixated on if the positive stimulus appeared there and to the other side key if it did not.

For at least six of the animals $(K, M, P, F, D$, and $R)$, it seems reasonable to say that the peak level of scanning occurred at about the point that learning neared the asymptote. The peak level of scanning for the remaining two animals ( $\mathrm{J}$ and $\mathrm{L}$ ), occurred later than that

Although learning was clearly accompanied by an increase in amount of scanning, there did not appear to be a consistent relationship between amount of scanning and rate or asymptote of learning. For example, we used the number of trials to reach a criterion of 19 trials correct during 20 consecutive trials as one index of rate of learning. We eliminated the data of animal $R$ from this analysis because of its atypical observing pattern, and added that of an 
animal that received identical treatment (except for a shorter overall period of training). ${ }^{1}$ Considering, first, only those animals that were given the squares problem we compared the amount of scanning of the three that reached criterion the most quickly $(\overline{\mathrm{X}}=$ 303 trials) with the three that reached it the most slowly $(\overline{\mathrm{X}}=573$ trials $)$. During the precriterion trials, the mean number of scans for the faster learners was .24 and for the slower, .39, while during the 20 criterion trials, the means for the two groups were .52 and .68 , respectively. Neither difference was significant as measured by the $t$ test. A comparable analysis of the data of animals $M$ and $P$ plus six animals that were given an irregular-pattern problem identical or similar to that given those two animals produced similar results. Taking another approach, on the squares problem, during the session in which the greatest amount of scanning occurred, the three animals with the lowest amount of scanning $(\overline{\mathrm{X}}=.67$ scans per trial) made $93 \%$ correct responses, while those with the highest amount of scanning $(\overline{\mathrm{X}}=$ 1.06) made $92 \%$.

Mean duration of the last fixation on a discriminative stimulus and mean duration of fixations on the discriminative stimuli preceding the last (fixations preceding) are shown in Figure 2 as a function of practice. The curves for average duration of the last fixation, although tending to show the same general increasing then decreasing trend that was seen for amount of scanning, were considerably more variable among the animals. The maximum duration of the last fixation and the maximum amount of scanning occurred during different sessions for six of the animals, with maximum duration occurring later for three of them $(M, D$, and R) and earlier for the other three (J, P, and L). In most cases, by the end of training the mean duration of the last fixation was close to what it was early in training. For purposes of statistical analysis, the average duration of the last fixation for each animal (except $\mathrm{K}$ ) was separated into the same six blocks as described for amount of scanning, except that the session of peak observing activity was now defined in terms of duration. The means of the six blocks were $238,306,374,409,357$, and $311 \mathrm{msec}$, respectively. Analysis of variance conducted on the log transformed values indicated that the effect of blocks was highly significant $[F(5,30)=6.63, \mathrm{p}<.001]$. The Newman-Keuls test indicated that the mean of Block 1 was significantly different from those of the other five blocks and that the mean of Block 4 (when duration was at the peak level) was significantly different from that of Block 6 as well as Block 1. There was no consistent pattern of change in average duration of the fixations preceding the last as a function of practice.

Since the latency of the instrumental choice

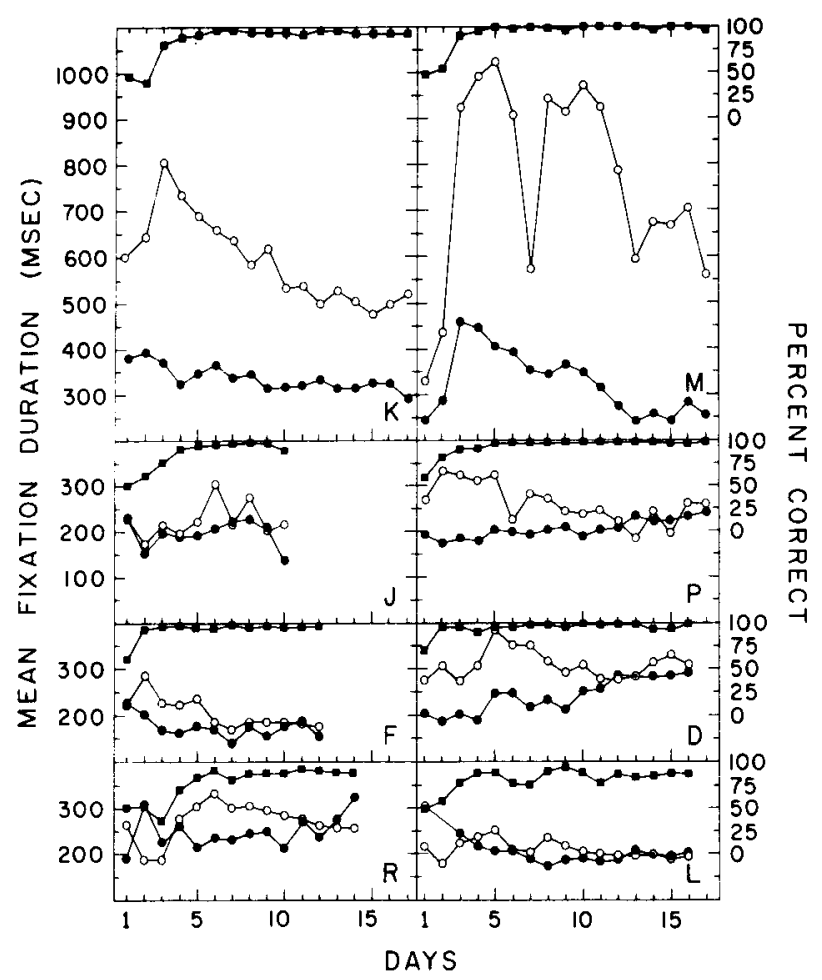

Figure 2. Mean duration of the last fixation on a discriminative stimulus (open circles) and of the fixations that preceded the last (closed circles and left ordinate) for each of the eight monkeys as a function of practice. Also shown is the percentage of correct responses (squares and left ordinate) as a function of days of practice.

response depends on, among other things, both how many times an animal looks at the discriminative stimuli and how long it does, it is not surprising that the shape of the curves relating latency and practice was also increasing, then decreasing. However, various characteristic features of the latency curves did not appear to be any better predicted by the features of the scanning curves than by those of the duration curves. This was true, for example, with respect to the session in which maximum latency occurred. Of the six animals whose maximum amount of scanning and maximum duration of last fixations occurred during different sessions, the session of maximum latency matched the session of maximum duration for two ( $\mathrm{J}$ and $\mathrm{R})$, the session of maximum scanning for two ( $M$ and $P$ ), and neither of these sessions for the remaining two (D and $L$ ).

\section{DISCUSSION}

Some of the conclusions of Tolman and Muenzinger about VTE during visual discrimination learning are supported by the present results, but others are not. In the present study, following the pattern seen with VTE, amount of scanning increased during learning 
and, for most animals, reached a peak at about the same time that learning reached the asymptote. Also, amount of scanning by nearly all the animals decreased following learning, producing the increasing then decreasing curve seen in the VTE studies when the problems were not difficult. Since the discriminative stimuli in the present study were not especially difficult to discriminate, as judged by the speed with which the animals learned the problems and the high levels of asymptotic performance, this last-mentioned outcome is consistent with the findings for VTE. Since essentially similar results were obtained in prior studies for both ocular (Geary \& Schrier, 1975) and nonocular observing (D'Amato et al., 1968), we conclude that the changes just described represent quite general characteristics of frequency of observing behavior during original learning of visual discrimination problems. On the other hand, contrary to what might have been expected on the basis of results for VTE, there was no clear-cut relationship between amount of scanning and speed or asymptotic level of learning. There has, in addition, been little indication of a relationship between the two in our previous work (Schrier \& Povar, 1978).

How might the obtained pattern of change in amount of scanning be explained? There have been two types of accounts of observing behavior that could be applied here. These are the conditioned reinforcement and information hypotheses (Fantino, 1977; Lieberman, 1972). Wyckoff (1952) proposed that observing responses are strengthened and maintained by conditioned reinforcement provided by the discriminative stimuli once they become differentially associated with primary reinforcement. Hence, Wyckoff would have predicted the increase in frequency of scanning that occurred during learning, but not the decrease. D'Amato et al. (1968) suggested that the decrease in frequency of nonocular observing that they found following acquisition of a visual discrimination could be the result of the delay in primary reinforcement produced by observing responses. Although such an explanation for the present results cannot be dismissed out of hand, the delays produced by the above-minimum fixations ( 250 to $350 \mathrm{msec}$ per fixation) are much smaller than those that are necessary to produce appreciable decreases in performance (D'Amato, 1973). Furthermore, in contrast to the usual nonocular observing response study, reinforcement in our eye-movement studies is not strictly dependent on cessation of the observing response, since the animals can and do make the choice response while they are observing.

The information explanations of observing behavior, employing, usually somewhat loosely, concepts of information theory, conceive of observing responses as representing a search for information in order to reduce uncertainty about experimental events and outcomes. This type of account has figured prominently in recent years in discussions of possible mechanisms of reinforcement of observing responses, especially in operant contexts where, for example, animals are given the opportunity to convert mixed to multiple schedules (Fantino, 1977; Lieberman, 1972). While it is possible that an information hypothesis could account for aspects of the present results, it is not clear how it could handle both the above-minimum amounts of scanning that develop with practice and the fact that they occur when the frequency of correct choice responses is near or at the maximum.

Another explanation of the ocular observing findings is that they are a product of motivational factors, perhaps an energization effect ("elation") related to the relatively sudden increase in frequency of reinforcement. The relative transience of the increase in observing is consistent with this type of hypothesis, as is the similar increase (discussed shortly) following sudden decreases in frequency of reinforcement ("frustration"). As D'Amato et al. (1968) have pointed out, if the increase in observing had a motivational basis, the effects should be nonspecific, influencing observing behavior on other problems or subproblems that the animals are receiving concomitantly, but the data indicate that this is not the case (D'Amato et al., 1968; Schrier \& Vaughan, 1973). Also, neither this hypothesis nor the others can explain why the peak level of observing, measured in terms of amount of scanning, often occurs on different days than the peak level measured in terms of duration of fixation.

None of the hypotheses mentioned so far appears to provide a very satisfactory explanation of the results and, hence, we are tempted to look further. ${ }^{2}$ In line with recent trends in interpretation of results of studies of eye movements of humans (Rayner, 1978; Senders, Fisher, \& Monty, 1978) as well as of monkeys (Geary \& Schrier, 1975; Schrier \& Povar, 1978), a promising avenue of approach would seem to be to view the changes in ocular observing as a function of practice as representing, at least in part, changes in information processing strategies. For example, the changes seen in the present work could reflect processes similar to those hypothesized by Nodine and Simmons (1974) to account for differences in amount of scanning by children of different ages who were discriminating between letter-like visual patterns. On the assumption that the present discriminations are based on the comparison of distinctive features, the monkeys, as appears to be the case with younger children, may initially develop a simple search strategy, selecting one or another distinctive feature of the first discriminative stimulus fixated on, doing the same with the second, and then 
testing for matching or contrast of the features. The visual search (scanning) continues until a contrasting feature of the positive stimulus is identified (in the present study, as in our previous ones, the animals almost always fixate on the positive stimulus before making a correct choice response). With practice, the animals might be developing a more efficient strategy, like that seen with older children, in which the search is limited to a particular distinctive feature characterizing the positive stimulus. One advantage that this account has over those already discussed is that it is better able to accommodate differences in the pattern of change in amount of scanning and in duration of fixation that occur as a function of practice, which, as the discussion to follow indicates, can be considerable. It can also easily accommodate the fact that the declines in high levels of scanning occur in some cases well past the point that choice behavior appears to have reached the asymptote. If this type of account is correct, we would expect smaller increases in amount of scanning during learning of brightness and color discrimination problems than for pattern discrimination problems.

Relatively transient increases in nonocular observing responses have been found (D'Amato et al., 1968) during extinction and during the presolution period of discrimination reversal learning when performance was at or below the chance level. Similar results have been obtained for ocular observing following reversal of a discrimination (Schrier \& Wing, 1973). The marked difference in the location of the maximum observing activity during original learning and during reversal learning, in the former case when frequency of reinforcement was very high or at the maximum level and in the latter when frequency of reinforcement was low, suggests that different processes may be influencing observing behavior in these different cases. D'Amato et al. (1968), in a variation of the information-type of hypothesis, proposed that their reversal and extinction findings could be a consequence of uncertainty resulting from nonreinforcement or inconsistent reinforcement. These data are clearly better handled by this type of account than by the information processing account proposed here for the case of original learning.

In the present experiment, the pattern of change in duration of the last fixation as a function of practice was considerably more variable among animals than was that for amount of scanning, although the increasing then decreasing trend was also present. The significant decline in duration of the last fixation after the peak level was reached to a level close to that seen during the initial phases of learning is noteworthy. It contrasts with our results for complex discrimination tasks (Geary \& Schrier, 1975) and for the learning set task (Schrier \& Povar, 1978) in which cases duration of the last fixation remained considerably above the level seen early in learning or remained at the maximum level even when training was continued well past the point that learning had reached the asymptote. On the basis of these experiments, we have suggested that the duration of the last fixation is particularly sensitive to the cognitive processing demands of discriminative tasks. Since the demands of the present task were certainly slight in this respect compared with those in the studies cited, the present results for last fixations are consistent with the previous ones. The present failure to find any consistent trend in duration of fixations preceding the last also contrasts with our findings for the learning set task (Schrier \& Povar, 1978).

The differences in results for amount of scanning and duration of both the last fixation and those preceding it support our conclusion (Geary \& Schrier, 1975; Schrier \& Povar, 1978) that these measures of ocular observing are reflecting different processes. As we have noted before, in the future, investigators dealing with observing behavior may have to distinguish more carefully than has been the case up to now between conclusions that apply to duration of observing and those that apply to frequency. Also, since, as already mentioned, latency of the choice response is influenced both by duration of fixation and amount of scanning, these latter measures may prove more useful for purposes of drawing inferences about information and cognitive processes underlying discriminative performance than is latency.

\section{REFERENCES}

Bower, G. H. Choice-point behavior. In R. R. Bush \& W. K. Estes (Eds.), Studies in mathematical learning theory. Stanford: Stanford University Press, 1959.

Bower, G. H. Response strengths and choice probability: A consideration of two combination rules. In E. Nagel, P. Suppes, \& A. Tarski (Eds.), Proceedings of the International Congress on Logic, Methodology and the Philosophy of Science, 1960. Stanford: Stanford University Press, 1962.

D'Amato, M. R., Etrin, M., \& Fazzaro, J. Cue-producing behavior in the capuchin monkey during reversal, extinction, acquisition and overtraining. Journal of the Experimental Analysis of Behavior, 1968, 11, 425-434.

D'Amato, M. R. Delayed matching and short-term memory in monkeys. In G. Bower (Ed.), The psychology of learning and motivation (Vol. 7). New York: Academic Press, 1973.

FAnTino, E. Conditioned reinforcement: Choice and information. In W. K. Honig \& J. E. R. Staddon (Eds.), Handbook of operant behavior. Englewood Cliffs, N.J: Prentice-Hall, 1977.

GeARY, N. D., \& Schrier, A. M. Eye movements of monkeys during performance of ambiguous cue problems. Animal Learning \& Behavior, 1975, 3, 167-171.

Geier, F. M., Levin, M., \& Tolman, E. C. Individual differences in emotionality, hypothesis formation, vicarious trial and error, and visual discrimination learning in rats. Comparative Psychology Monographs, 1941, 17(No. 3).

Goss, A. E., \& Wischner, G. J. Vicarious trial and error and related behavior. Psychological Bulletin, 1956, 53, 35-54. 
Lieberman, D. A. Observing behavior in mixed schedules by monkeys (Macaca mulatta).Learning and Motivation, 1972, 3, 341-358.

Muenzinger, K. F. Vicarious trial and error at a point of choice: I. A general survey of its relation to learning efficiency. Journal of Genetic Psychology, 1938, 53, 75-86.

Nodine, C. F., \& Simmons, F. G. Processing distinctive features in the differentiation of letterlike symbols. Journal of Experimental Psychology, 1974, 103, 21-28.

RAYNER, $K$. Eye movements in reading and information processing. Psychological Bulletin, 1978, 85, 618-660.

Schrier, A. M., Povar, M. L., \& Vaughan, J. Measurement of eye orientation of monkeys during visual discrimination. Behavior Research Methods \& Instrumentation, 1970, 2, 55-62.

Schrier, A. M., \& Povar, M. L. Eye movements of monkeys during learning set formation. Science, 1978, 199, 1362-1364.

Schrie R, A. M., \& Wing, T. G. Eye movements of monkeys during brightness discrimination and discrimination reversal. Animal Learning \& Behavior, 1973, 1, 145-150.

Schrier, A. M., \& Vaughan, J. Eye movements of monkeys during learning of color and form discrimination problems involving reversal and nonreversal shifts. Primates, 1973, 14, 161-178.

Senders, J. W., Fisher, D. F., \& Monty, R. A. Eye movements and the higher psychological processes. Hillsdale, N.J: Erlbaum, 1978.

Spence, K. Behavior theory and learning. Englewood Cliffs, N.J: Prentice-Hall, 1960.

Tolman, E. C. The determiners of behavior at the choice point. Psychological Review, 1938, 45, 1-41.

Tolman, E. C. Cognitive maps in rats and men. Psychological Review, 1948, 55, 189-208.

Tolman, E. C., \& Minium, E. VTE in rats: Overtraining and difficulty of discrimination. Journal of Comparative Psychology, 1942, 34, 301-306.

VAUGHAN, J. On-line, real-time recording of eye orientation using the corneal reflection method. Behavior Research Methods \& Instrumentation, 1975, 7, 211-214.
Wyckoff, L. B., JR. The role of observing responses in discrimination learning: Part I. Psychological Review, 1952, 59, 431-442.

\section{NOTES}

1. This animal also showed the increasing-decreasing pattern of change in amount of scanning with practice. Its curve was most like that of animal $\mathrm{J}$, with the peak occurring on the 3rd day of asymptotic performance, at which time it was making $98 \%$ correct responses.

2. We might also mention that Bower (1959) and Spence (1960, chap. 22), among others, formulated random walk VTE models of choice behavior. If, as seems reasonable under the conditions of most of the studies in which the increasing then decreasing VTE or observing response function has been obtained, it is assumed that the initial probabilities of response to the discriminative stimuli (once oriented to) are about equal and .5 or lower and diverge in the appropriate directions with practice, then these models predict that the frequency of observing will decrease monotonically from a relatively high initial level to .5 as frequency of correct responses increases from the chance level (cf. Bower, 1962). It does seem possible for the models to predict a function of the type reported here with some special kinds of changes in the response probabilities as a function of practice. One such type of change would be where the initial probabilities are equal and high, then there is a drop in the probability of responding to the negative stimulus, with little or no rise in the probability of responding to the positive, and, finally, there follows a continued drop in the former probability and a rise in the latter. The models seem to handle more straightforwardly the function found during the early part of reversal training, discussed later in the paper, where the initial probability of response to the incorrect stimulus (formerly correct) is higher than that to the correct one (Bower, 1962).

(Received for publication July 12, 1978; revision accepted October 12,1978 .) 